\title{
Consolidated bioprocessing of transgenic switchgrass by an engineered and evolved Clostridium thermocellum strain
}

Kelsey L Yee ${ }^{1,2}$, Miguel Rodriguez $\mathrm{Jr}^{1,2}$, Olivia A Thompson ${ }^{1,2}$, Chunxiang Fu ${ }^{3,4}$, Zeng-Yu Wang ${ }^{2,3}$, Brian H Davison ${ }^{1,2^{*}}$ and Jonathan R Mielenz ${ }^{1,2,5}$

\begin{abstract}
Background: Switchgrass is an abundant and dedicated bioenergy feedstock, however its inherent recalcitrance is one of the economic hurdles for producing biofuels. The downregulation of the caffeic acid O-methyl transferase (COMT) gene in the lignin pathway of switchgrass reduced lignin content and S/G ratio, and the transgenic lines showed improved fermentation yield with Saccharomyces cerevisiae and wild-type Clostridium thermocellum (ATCC 27405) in comparison to the wild-type switchgrass.

Results: Here we examine the conversion and yield of the COMT transgenic and wild-type switchgrass lines with an engineered and evolved C. thermocellum (M1570) strain. The fermentation of the transgenic switchgrass by M1570 had superior conversion relative to the wild-type control switchgrass line with an increase in conversion of approximately $20 \%$ and ethanol being the primary product accounting for $90 \%$ of the total metabolites measured by HPLC analysis.

Conclusions: The engineered and evolved C. thermocellum M1570 was found to respond to the apparent reduced recalcitrance of the COMT switchgrass with no substrate inhibition, producing more ethanol on the transgenic feedstock than the wild-type substrate. Since ethanol was the main fermentation metabolite produced by an engineered and evolved C. thermocellum strain, its ethanol yield on a transgenic switchgrass substrate (gram/gram $(\mathrm{g} / \mathrm{g})$ glucan liberated) is the highest produced thus far. This result indicates that the advantages of a modified feedstock can be combined with a modified consolidated bioprocessing microorganism as anticipated.
\end{abstract}

Keywords: Transgenic, Switchgrass, Metabolic engineering, Clostridium thermocellum, Consolidated bioprocessing, Feedstock, Cellulosic ethanol

\section{Background}

Lignocellulosic biomass is an abundant feedstock but its inherent recalcitrance towards conversion is one of the major economic hurdles to producing biofuels [1,2]. Lignin is a major component of plant cell walls and a contributor to recalcitrance by negatively impacting enzymatic hydrolysis of cellulose and hemicellulose to fermentable sugars $[3,4]$. Genetic engineering of feedstocks to reduce lignin content and/or improve composition has been

\footnotetext{
* Correspondence: davisonbh@ornl.gov

${ }^{1}$ Biosciences Division, Oak Ridge National Laboratory, Oak Ridge, TN 37831-6341, USA

2BioEnergy Science Center, Oak Ridge National Laboratory, Oak Ridge, TN 37831-6037, USA

Full list of author information is available at the end of the article
}

shown to increase the accessibility of the cellulose and hemicellulose to enzymatic hydrolysis and as a result improve fermentation yield [5-11].

Although variation exists among these processes, the majority of lignocellulosic feedstocks to ethanol bioconversion schemes have four primary objectives: reduction of biomass particle size, biomass pretreatment, hydrolysis of carbohydrate polymers to fermentable sugars, and fermentation of these sugars to ethanol. Consolidated bioprocessing (CBP) approaches combine enzyme production, substrate hydrolysis, and fermentation into one process. Despite continued reductions in the cost of hydrolytic enzymes, CBP remains an attractive alternative due to the potential of decreasing costs associated 
with reducing unit operations and exogenous enzyme supplementation [12-14].

Clostridium thermocellum is a thermophilic and cellulolytic Gram-positive bacterium that is considered a consolidated bioprocessing candidate because of its ability to grow rapidly on native crystalline cellulose without the addition of exogenous enzymes. Moreover, C. thermocellum fermentations showed the utilization of up to $75 \%$ of thecellulose contained in pretreated biomass sources [7,15-17]. However, the wild-type strain has a mixed-product fermentation profile consisting of lactic acid, formic acid, acetic acid, and ethanol as well as carbon dioxide and hydrogen. As a result strain engineering approaches are needed to increase the yield of ethanol and minimize the production of the other metabolites [18]. Argyros et al. [19] developed a strain of C. thermocellum (M1570) that was evolved for growth and contained deletions in the acetic and lactic acid pathways $(\Delta$ hpt $\Delta$ ldh $\Delta$ pta). The strain produced ethanol in a 40:1 ratio with respect to residual organic acids in actively $\mathrm{pH}$ controlled Avicel fermentations.

Here we expand on the previous work of Fu et al. [7] and Yee et al. [8] where they showed that downregulation of the caffeic acid 3-O-methyltransferase (COMT) gene in switchgrass reduced recalcitrance and improved microbial bioconversion yield regardless of pretreatment condition for simultaneous saccharification and fermentation (SSF) with S. cerevisiae $[7,8]$. In contrast to yeastbased SSF, C. thermocellum (ATCC 27405) fermentations demonstrated sensitivity to the COMT transgenic biomass. It was postulated that additional soluble lignin pathway-derived constituents resulting from the COMT gene disruption measured by GC-MS in the fermentation broth may have led to the sensitivity and/or inhibition $[8,20]$. The fermentation sensitivity was not observed in the fermentation of the wild-type switchgrass line. This inhibition can be eliminated by extensive washing or hot water extraction, which allowed C. thermocellum to have a superior yield of fermentation products with the transgenic substrate versus wild-type line biomass, exceeding the yeast-based SSF yield $[7,8]$. In this study we fermented the less recalcitrant transgenic COMT switchgrass with the recombinant C. thermocellum strain M1570 developed by Argyros et al. [19]. We show that the fermentation of the transgenic biomass had superior conversion in comparison to the wild-type switchgrass and the primary fermentation end-product metabolite is ethanol at a 9:1 ratio of ethanol to acetic acid.

\section{Results}

From the previous studies, fermentation of dilute-acid pretreated and extensively washed switchgrass had higher conversions than hot water or no pretreatment $[7,8]$. As a result the biomass was dilute acid pretreated and hot water extracted prior to fermentation. The carbohydrate composition of the biomass after dilute acid pretreatment was primarily glucan from cellulose measured by the quantitative saccharification assay. The dilute acid pretreatment solubilized the hemicellulose and the extensive washing removed soluble compounds and extractives.

The mutant (M1570) and wild-type (DSM 1313) C. thermocellum fermentations of the pretreated and washed transgenic (T1-3-TG) and wild-type control (T1-3-WT) switchgrass lines were monitored over time by tracking weight loss to gaseous products through periodic venting of the tared serum bottle. At 145 hours the weight loss stabilized indicating that the fermentations had ceased. Analysis of the endpoint fermentation broth was performed using HPLC measuring soluble sugars (glucose, cellobiose, arabinose, and xylose) and the primary fermentation metabolites for $C$. thermocellum (acetic acid, lactic acid, ethanol, and formic acid). The fermentation broths did not contain formic acid, cellobiose, arabinose, or xylose as measured by HPLC. The endpoint conversion was reported as $\mathrm{mg} / \mathrm{g}$ glucan loaded for both metabolites and soluble but unfermented glucose. The transgenic line had greater conversion to fermentation metabolites than the wild-type by 11 and 27\% for DSM 1313 and M1570, respectively (Figure 1, Table 1, and Additional file 1: Table S1). Moreover, the product profiles showed that ethanol was approximately $90 \%$ of the total metabolite conversion for fermentations with M1570 on either the transgenic or the wild-type switchgrass $(1.7 \mathrm{~g} / \mathrm{L}$ and $1.2 \mathrm{~g} / \mathrm{L}$, respectively). In contrast, the DSM 1313 fermentations produced acetic acid and ethanol at a ratio of approximately 2:1 for both transgenic and wild-type switchgrass with minimal amounts of lactic acid production.

The T1-3-TG fermentation with M1570 had a conversion of $0.19 \mathrm{~g} / \mathrm{g}$ glucan loaded or a yield of $0.27 \mathrm{~g} / \mathrm{g}$ glucan liberated (Additional file 1: Table S1 and Additional file 2: Table S2), which is equivalent to the $0.27 \mathrm{~g} / \mathrm{g}$ yield of ethanol achieved in the $\mathrm{pH}$ controlled Avicel fermentations by Argyros et al. [19]. The mutant and the wild-type C. thermocellum strains had similar conversions for fermentations on the same biomass line but significantly different metabolite ratios (Figure 1 and Additional file 1: Table S1). There were not substantial concentrations of liberated but unfermented sugars in the broth in the M1570 strain fermentations, but there was a slight increase of a 2.4-fold in residual, soluble, unfermented glucose in the DSM 1313 strain fermentation of the T1-3-TG in comparison to the T1-3-WT (Figure 1 and Additional file 1: Table S1). This could be due to the fact that these fermentations were heavily buffered to $\mathrm{pH} 7.0$, but not actively $\mathrm{pH}$ controlled while the optimal growth range is above 6.2. The endpoint $\mathrm{pH}$ was approximately 6.3 for the M1570 fermentations and approximately 5.8 for the wild type C. thermocellum fermentations which is below the optimal growth range. Previous results showed an 18\% 


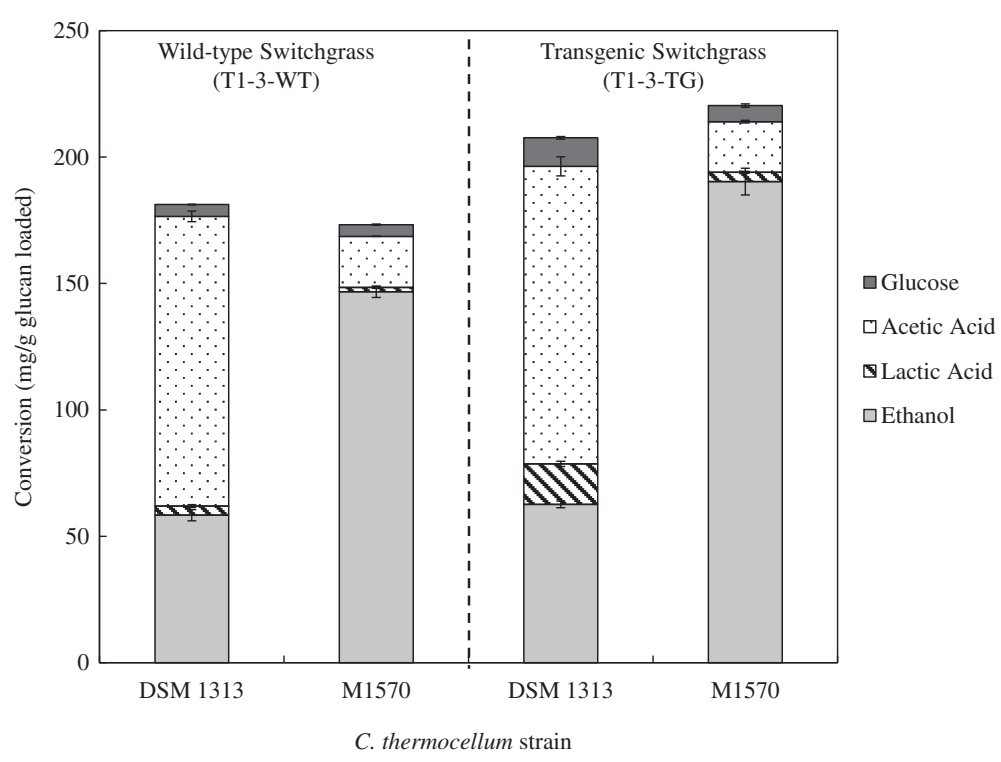

Figure 1 Conversion (mg/g glucan loaded) for C. thermocellum mutant M1570 and wild-type DSM 1313 strains on both transgenic (T1-3-TG) and wild-type (T1-3-WT) switchgrass. The standard deviation is from the average of triplicate buffered serum bottle fermentations. The light gray bars represent ethanol, the dashed bar represents lactic acid, the dotted bar represents acetic acid, and the dark gray bar represents soluble unfermented glucose.

improvement for fermentations with wild-type C. thermocellum (ATCC 27405) in actively pH controlled fermentations on COMT switchgrass [7]. Here we only show an $11 \%$ improvement in a buffered batch system; the residual, soluble, unfermented glucose is reported and cannot account for this difference. The $\mathrm{pH}$ and headspace effects may account for this difference in conversion of the transgenic biomass compared to the wild-type line. In addition to conversion, yield $(\mathrm{mg} / \mathrm{g}$ glucan liberated) was evaluated for the fermentations described above. The glucan liberated from the biomass was determined by analysis of the solid fermentation residues using a quantitative saccharification assay for carbohydrate composition. The yield was calculated based on the assumption that all glucan liberated was converted to fermentation metabolites measured by HPLC (Table 1). A change in microbe from the wildtype (DSM 1313) to the mutant (M1570) significantly changed the product ratio from approximately 2:1 to $1: 9$ acetic acid to ethanol, regardless of wild-type versus transgenic switchgrass, but not the overall yield. A change from wild-type to transgenic switchgrass, improved the accessibility and conversion of the glucan, regardless of microbe, but not the overall fermentation yield. Moreover, regardless of the $C$. thermocellum strain, the transgenic switchgrass had more glucan liberated indicating that the cellulose was more easily accessible. Analysis of endpoint fermentation metabolites and unfermented soluble glucose indicated a total mass balance closure of approximately $60 \%$ for the above fermentations. This calculation was based on a stoichiometric ratio of 1:1 for ethanol to $\mathrm{CO}_{2}$ and acetic acid to $\mathrm{CO}_{2}$. In addition, it does not include maintenance, cell density, and protein production. The mass balance remains open and that calculation coupled with low yields suggests that carbon may be diverted through alternate pathways to side products that were not measured.

Table 1 Endpoint fermentation analysis of $C$. thermocellum strains on transgenic and wild-type switchgrass

\begin{tabular}{lcccc}
\hline $\begin{array}{l}\text { Strain/ } \\
\text { Switchgrass }\end{array}$ & $\begin{array}{c}\text { Total products (acetic acid + lactic } \\
\text { acid + ethanol) } \mathbf{( m g )}\end{array}$ & $\begin{array}{c}\text { Glucan liberated from } \\
\text { biomass }(\mathbf{m g})\end{array}$ & $\begin{array}{c}\text { Yield (mg total products/g } \\
\text { glucan liberated) }\end{array}$ & $\begin{array}{c}\text { Conversion (mg total } \\
\text { products/g glucan loaded) }\end{array}$ \\
\hline DSM 1313/ & $73.1 \pm 2.0$ & $226 \pm 5$ & $324 \pm 6$ & $178 \pm 2$ \\
T1-3-WT & $87.3 \pm 3.0$ & $264 \pm 16$ & $332 \pm 26$ & $198 \pm 6$ \\
DSM 1313/ & $69.5 \pm 1.2$ & & $300 \pm 17$ & $170 \pm 3$ \\
T1-3-TG & & $232 \pm 13$ & $310 \pm 16$ & $216 \pm 6$ \\
M1570/ & $95.8 \pm 2.6$ & $309 \pm 13$ & & \\
T1-3-WT & & & & \\
M1570/ & & & & \\
T1-3-TG & & & & \\
\hline
\end{tabular}




\section{Discussion}

Transgenic feedstocks with reduced recalcitrance in combination with consolidated bioprocessing microbes have the potential to further reduce the cost of biofuels by eliminating or drastically reducing the requirement of exogenous hydrolytic enzymes. Therefore we examined the fermentation performance of the $C$. thermocellum M1570 strain on transgenic COMT switchgrass. We showed that fermentations with either the wild-type or mutant $C$. thermocellum strain on the transgenic switchgrass had improved conversion (mg/g glucan loaded), total products, and glucose liberated in comparison to the wildtype switchgrass indicating that the transgenic switchgrass was more easily digested. Moreover, the conversion for the M1570 strain was $90 \%$ ethanol with minimal amounts of the side products lactic and acetic acid regardless of switchgrass line. Fermentations of transgenic or wild-type switchgrass by either $C$. thermocellum strain gave comparable total end-product fermentation yields $(\mathrm{mg} / \mathrm{g}$ glucan liberated), however with significantly different metabolite ratios. The similar yields and minimal residual unfermented carbohydrates in the fermentation broth indicate that the glucan liberated and utilized for measured endpoint fermentation products was similar for both strains.

The mass balance remains open for these fermentations where only endpoint metabolites and soluble but unfermented glucose is considered. The mass balance closure was approximately $60 \%$ under the assumption that glucan liberated was converted to fermentation metabolites and $\mathrm{CO}_{2}$ was estimated using the stoichiometric ratio of 1:1 for both ethanol to $\mathrm{CO}_{2}$ and acetic acid to $\mathrm{CO}_{2}$. In addi tion, both $C$. thermocellum strains had low endpoint fermentation yields and this may be in part be due to carbon flow through alternate pathways. Argyros et al. [19] and van der Veen et al. [21] both hypothesized that the disruption of fermentation metabolic pathways can lead to a redox imbalance and metabolism bottlenecks. They both showed that there is an accumulation of central metabolites, such as pyruvate, in their deletion strains. Moreover, van der Veen et al. [21] showed that their evolved $\Delta$ hpt $\Delta$ ldh $\Delta$ pta mutant channeled $17 \%$ of the available carbon to secreted amino acids. The open mass balance and low yields from these fermentations suggests that there is a redox-imbalance in $\Delta$ hpt $\Delta$ pta $\Delta$ ldh mutant strain and the carbon is diverted through alternate pathways. This issue may be potentially solved through further study of intracellular redox state and metabolic pathways leading to alternate engineering strategies [22-24].

\section{Conclusions}

This set of fermentations establishes a baseline for CBP conversion of transgenic switchgrass with a genetically engineered and evolved C. thermocellum strain (M1570) that produces primarily ethanol. The mutant strain responded to the reduced recalcitrant transgenic COMT switchgrass with the same trend as the wild-type $C$. thermocellum. The ethanol yield for the mutant strain was significantly higher for the fermentation of the transgenic versus the wild-type control switchgrass. These results give additional support toward the potential of transgenic COMT switchgrass as a biomass feedstock line with reduced recalcitrance and improved fermentation yield. With further understanding of intracellular metabolism and redox state leading to engineered strains with improved ethanol yield, rate, and titer, $\mathrm{CBP}$ with $C$. thermocellum mutants in combination with biomass with reduced recalcitrance have the potential to further reduce the costs of cellulosic ethanol.

\section{Materials and methods}

\section{Growth and harvesting conditions for transgenic and} control plant material

COMT down-regulated transgenic and wild-type control switchgrass (Panicum virgatum L.) lines were generated by the Samuel Roberts Noble Foundation as previously described in Fu et al. [7].

\section{Pretreatment}

The biomass was milled in a Wiley mill using a 20 mesh screen (Thomas Scientific, Swedesboro, NJ, USA). Dilute acid pretreatment was performed as described previously in Fu et al. [7] and Yee et al. [8]. Briefly, the biomass was soaked overnight in $0.5 \% \mathrm{H}_{2} \mathrm{SO}_{4}$ and loaded at a ratio of $2.5 \mathrm{~g}$ dry per Hastelloy steel tubular pretreatment reactor (Industrial Alloys Plus Inc., Utica, KY, USA). The reactors were preheated in boiling water for 2 minutes and then transferred to a fluidized sand bath (Omega FSB1:Techne Co., Cole Parmer, Court Vernon Hills, IL, USA) at $180^{\circ} \mathrm{C}$ for 7.5 minutes $[7,25]$. The reactors were cooled by quenching in an ice bath. The biomass was washed with $100 \mathrm{~mL}$ Milli-Q water (Millipore Corporation, Billerica, MA, USA) per gram dry biomass and then subjected to a hot water extraction to remove inhibitory water soluble compounds and washed a second time as described previously by Yee et al. [8]. The solid residual biomass was stored at $-20^{\circ} \mathrm{C}$ until fermentation.

\section{Consolidated bioprocessing conversion}

The Lee R. Lynd Lab at Dartmouth College (Hanover, $\mathrm{NH}$, USA) generously donated the $C$. thermocellum DSM 1313 strain and the Mascoma Corporation (Lebanon, NH, USA) provided the $C$. thermocellum M1570 strain. The M1570 strain was developed in the C. thermocellum DSM $1313 \Delta$ hpt background strain. Lactate dehydrogenase (Ldh) and phosphotransacetylase (Pta) genes were deleted and the $\Delta$ hpt $\Delta$ ldh $\Delta$ pta mutant strain was transferred in batch culture for 2,000 hours and a stable strain was achieved [19]. All CBP fermentations were cultivated at 
$55^{\circ} \mathrm{C}$ and underwent orbital shaking at $125 \mathrm{rpm}$ in defined no-yeast extract media for thermophilic clostridia (MTC) [26]. Bottles were loaded with $0.75 \mathrm{~g}$ of biomass on a dry basis, autoclaved for 30 minutes for sterilization purposes, and a $2.0 \% \mathrm{vol} / \mathrm{vol}$ inoculum was used for a final volume of $50 \mathrm{~mL}$. The inoculum was grown in $125 \mathrm{~mL}$ anaerobic serum bottles with $50 \mathrm{~mL}$ of MTC media and a carbon source of $5.0 \mathrm{~g} / \mathrm{L}$ Avicel (FMC BioPolymer, Newark, DE, USA) at $125 \mathrm{rpm}$ and $55^{\circ} \mathrm{C}$. Samples were not removed from the serum bottles during fermentation; instead weight loss was used to monitor the progress of the fermentation $[7,8,10,27]$.

\section{Analytical methods}

Endpoint fermentation broth samples were analyzed for metabolites (acetic acid, lactic acid, and ethanol) and residual carbohydrates (cellobiose, glucose, xylose, arabinose) using a high performance liquid chromatography (HPLC) LaChrom Elite ${ }^{\text {tm }}$ system (Hitachi High Technologies America Inc., Schaumburg, IL, USA) equipped with a refractive index detector (model L-2490). The products and carbohydrates were separated using an Aminex ${ }^{\mathrm{Tx}}$ HPX87H column (Bio-Rad Laboratories Inc., Hercules, CA, USA) at a flow rate of $0.5 \mathrm{~mL} / \mathrm{min}$ of $5.0 \mathrm{mM}$ sulfuric acid and a column temperature of $60^{\circ} \mathrm{C}[7,8,16]$.

Pretreated and washed biomass and fermentation residues were analyzed for carbohydrate composition using a quantitative saccharification assay NREL/TP-510-42618 and HPLC method NREL/TP-510-42623. Briefly, the samples were analyzed for carbohydrate composition using a high performance liquid chromatography (HPLC) LaChrom Elite $^{\text {rm }}$ system (Hitachi High Technologies America Inc.) equipped with a refractive index detector (model L-2490) and a UV-Vis detector (model L-2420). The carbohydrates (glucose, xylose, galactose, mannose, and arabinose) and pentose and hexose sugar degradation products (furfural and 5-hydroxy methyl furfural) were separated using an Aminex ${ }^{\text {Tw }}$ HPX-87P column (Bio-Rad Laboratories Inc.), at a $0.6 \mathrm{~mL} / \mathrm{min}$ flow rate of water and a column temperature of $80^{\circ} \mathrm{C}[7,8]$.

C. thermocellum utilizes hexose sugars, and after dilute acid pretreatment and extensive washing the hexose sugar content of the biomass was primarily glucan from cellulose because the dilute acid pretreatment solubilized the hemicellulose and the extensive washing removed extractives and soluble components. Analysis of the carbohydrate composition post-pretreatment and washing showed negligible amounts of mannose, arabinose, and xylose. Both prior to and post-fermentation the carbohydrate content of the biomass was determined by the quantitative saccharification assay. The conversion was calculated based on the initial glucan content of the biomass loaded and the yield was calculated based on glucan liberated from the biomass. Both conversion and yield are calculated under the assumption that all available glucan was converted to fermentation products.

\section{Additional files}

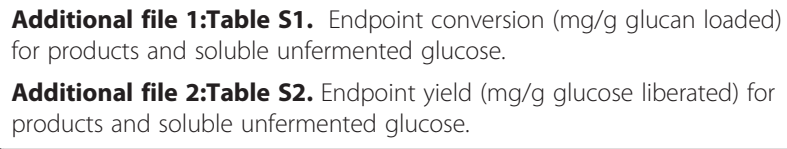

Additional file 2:Table S2. Endpoint yield ( $\mathrm{mg} / \mathrm{g}$ glucose liberated) for products and soluble unfermented glucose.

\section{Abbreviations}

ATCC: American type culture collection; CBP: Consolidated bioprocessing; COMT: Caffeic acid 3-O-methyltransferase; CTRL: Control; DA: Dilute acid pretreatment; HPLC: High performance liquid chromatography; HW: Hot water; SSF: Simultaneous saccharification and fermentation; T1: Generation one; TG: Transgenic; WT: Wild-type.

\section{Competing interests}

The authors declare that they have no competing interests.

\section{Authors' contributions}

KLY planned the work, conducted the experiments and wrote the manuscript. MRJr assisted in data acquisition and analysis and edited the manuscript. OAT helped run the fermentations and edited the manuscript. CF and ZYW produced and supplied the transgenic switchgrass material and edited the manuscript. JRM helped plan the experiments and edited the manuscript. BHD helped interpret the results and plan and edit the manuscript. All authors have read and approved the final manuscript.

\section{Acknowledgements}

We would like to thank Mrs Choo Y Hamilton for her technical assistance and support to this project. We would also like to thank D Aaron Argyros for his assistance and support and the Mascoma Corporation for providing the C. thermocellum M1570 strain. This research was funded by the Bioenergy Science Center (BESC) under grant ERKP695, which is a US Department of Energy (DOE) Bioenergy Research Center supported by the Office of Biological and Environmental Research in the DOE Office of Science. This manuscript has been authored by a contractor of the US Government under contract DE-AC05-000R22725.

\section{Author details}

${ }^{1}$ Biosciences Division, Oak Ridge National Laboratory, Oak Ridge, TN 37831-6341, USA. ${ }^{2}$ BioEnergy Science Center, Oak Ridge National Laboratory, Oak Ridge, TN 37831-6037, USA. ${ }^{3}$ Forage Improvement Division, The Samuel Roberts Noble Foundation, Ardmore, OK 73401, USA. ${ }^{4}$ Current address: Qingdao Institute of Bioenergy and Bioprocess Technology, CAS, No.189 Songling Rd, Qingdao City, Shandong Province 266101, People's Republic of China. ${ }^{5}$ White Cliff Biosystems, Rockwood, TN 37854, USA.

Received: 14 January 2014 Accepted: 8 May 2014

Published: 22 May 2014

\section{References}

1. Himmel ME, Ding SY, Johnson DK, Adney WS, Nimlos MR, Brady JW, Foust TD: Biomass recalcitrance: engineering plants and enzymes for biofuels production. Science 2007, 315:804-807.

2. Raguaskas AJ, Williams CK, Davison BH, Britovsek G, Cairney J, Eckert CA, Frederick WJ Jr, Hallett JP, Leak DJ, Liotta CL, Mielenz JR, Murphy R, Templer $\mathrm{R}$, Tschaplinski TJ: The path forward for biofuels and biomaterials. Science 2006, 311:484-489.

3. Li X, Weng J-K, Chapple C: Improvement of biomass through lignin modification. Plant J 2008, 54:569-581.

4. Grabber JH, Mertens DR, Kim H, Funk C, Lu F, Ralph J: Cell wall fermentation kinetics are impacted more by lignin content and ferulate cross-linking than by lignin composition. J Sci Food Agric 2009, 89:122-129.

5. Chen F, Dixon RA: Lignin modification improves fermentable sugar yields for biofuel production. Nature Biotechnol 2007, 25:759-761. 
6. Hisano H, Nandakumar R, Wang Z-Y: Genetic modification of lignin biosynthesis for improved biofuel production. In Vitro Cell Dev Biol Plant 2009, 45:306-307.

7. Fu C, Mielenz JR, Xiao X, Ge Y, Hamilton CY, Rodriguez M Jr, Chen F, Foston M, Ragauskas AJ, Bouton J, Dixon RA, Wang Z-Y: Genetic manipulation of lignin reduces recalcitrance and improves ethanol production from switchgrass. Proc Natl Acad Sci U S A 2011, 108:3803-3808.

8. Yee KL, Rodriguez M Jr, Tschaplinski TJ, Engle NL, Martin MZ, Fu C, Wang Z-Y, Hamilton-Brehm SD, Mielenz JR: Evaluation of the bioconversion of genetically modified switchgrass using simultaneous saccharification and fermentation and a consolidated bioprocessing approach. Biotechnol Biofuels 2012, 5:81.

9. Shen $\mathrm{H}, \mathrm{He} X$, Poovaiah CR, Wuddineh WA, Ma J, Mann DGJ, Wang H, Jackson L, Stewart CN Jr, Chen F, Dixon RA: Functional characterization of the switchgrass (Panicum virgatum) R2R3-MYB transcription factorPvMYB4 for improvement of lignocellulosic feedstocks. New Phytol 2012, 193:121-136.

10. Shen H, Poovaiah CR, Ziebell A, Tschaplinski TJ, Pattathil S, Gjersing E, Engle NL, Katahira R, Pu Y, Sykes R, Chen F, Ragauskas AJ, Mielenz JR, Hahn MG, Davis M, Stewart CN Jr, Dixon RA: Enhanced characteristics of genetically modified switchgrass (Panicum virgatum L.) for high biofuel production. Biotechnol Biofuels 2013, 6:71

11. Simmons BA, Loqué $D$, Ralph J: Advances in modifying lignin for enhanced biofuel production. Curr Opin Plant Biol 2010, 13:312-319.

12. Lynd LR, Weimer PJ, van ZyI WH, Pretorius IS: Microbial cellulose utilization: fundamentals and biotechnology. Microbiol Mol Biol Rev 2002, 66:506-577.

13. Lynd LR, Van Zyl WH, McBride JE, Laser M: Consolidated bioprocessing of cellulosic biomass: an update. Curr Opin Biotecnol 2005, 16:577-583.

14. Olson DG, McBride JE, Shaw JA, Lynd LR: Recent progress in consolidated bioprocessing. Curr Orin Biotechnol 2012, 23:396-405.

15. Shao X, Jin M, Guseva A, Liu C, Balan V, Hogsett D, Dale BE, Lynd L: Conversion of Avicel and AFEX pretreated corn stover by Clostridium thermocellum and simultaneous saccharification and fermentation: insights into microbial conversion of pretreated cellulosic biomass. Bioresourc Technol 2011, 102:8040-8045.

16. Raman B, Pan C, Hurst GB, Rodriguez M Jr, McKeown CK, Lankford PK, Samatova NF, Mielenz JR: Impact of pretreated switchgrass and biomass carbohydrates on Clostridium thermocellum ATCC 27405 cellulosome composition: a quantitative proteomic analysis. PLoS One 2009, 4:e5271.

17. Blumer-Schuette SE, Kataeva I, Westpheling J, Adams MWW, Kelly RM: Extremely thermophilic microorganisms for biomass conversion: status and prospects. Curr Opin Biotecnol 2008, 19:210-217.

18. Tripathi SA, Olson DG, Argyros DA, Miller BB, Barrett TF, Murphy DM, McCool JD, Warner AK, Rajgarhia VB, Lynd LR, Hogsett DA, Caiazza NC: Development of pyrF-based genetic system for targeted gene deletion in Clostridium thermocellum and creation of a pta mutant. Appl Environ Microbiol 2010, 76:6591-6599.

19. Argyros DA, Tripathi SA, Barrett TF, Rogers SR, Feinberg LF, Olson DG, Foden JM, Miller BB, Lynd LR, Hogsett DA, Caiazza NC: High ethanol titers from cellulose by using metabolically engineered thermophilic, anaerobic microbes. Appl Environ Microbiol 2010, 77:8288-8294

20. Tschaplinski TJ, Standaert RF, Engle NL, Martin MZ, Sangha AK, Parks JM, Smith JC, Samuel R, Jiang N, Pu Y, Ragauskas AJ, Hamilton CY, Fu C, Wang Z-Y, Davison BH, Dixon RA, Mielenz JR: Down-regulation of the caffeic acid O-methyltransferase gene in switchgrass reveals a novel monolignol analog. Biotechnol Biofuels 2012, 5:71.

21. van der Veen D, Lo J, Brown SD, Johnson CM, Tschaplinski TJ, Martin M, Engle NL, van den Berg RA, Argyros DA, Caiazza NC, Guss AM, Lynd LR: Characterization of Clostridium thermocellum strains with disrupted fermentation end-product pathways. J Ind Microbio Biotechnol 2013, 40:725-734

22. Li HF, Knutson BL, Nokes SE, Lynn BC, Flythe MD: Metabolic control of Clostridium thermocellum via inhibition of hydrogenase activity and the glucose transport rate. Appl Microbiol Biotechnol 2012, 93:1777-1784.

23. Roberts SB, Gowen CM, Brooks JP, Fong SS: Genome-scale metabolic analysis of Clostridium thermocellum for bioethanol production. BMC Syst Biol 2010, 4:31.

24. Deng Y, Olson DG, Zhou J, Herring CD, Shaw AJ, Lynd LR: Redirecting carbon flux through exogenous pyruvate kinase to achieve high ethanol yields in Clostridium thermocellum. Metab Eng 2013, 15:151-158.
25. Yang B, Wyman CE: Dilute Acid and Autohydrolysis Pretreatment. In Biofuels. Edited by Mielenz JR. New York: Humana; 2009:103-114.

26. Kridelbaugh DM, Nelson J, Engle NL, Tschaplinski TJ, Graham DE: Nitrogen and sulfur requirements for Clostridium thermocellum and Caldicellulosiruptor bescii on cellulosic substrates in minimal media. Bioresour Technol 2013, 130:125-135.

27. Mielenz JR, Bardsley JS, Wyman CE: Fermentation of soy bean hulls to ethanol while preserving protein value. Bioresource Technol 2009, 100:3532-3539.

doi:10.1186/1754-6834-7-75

Cite this article as: Yee et al:: Consolidated bioprocessing of transgenic switchgrass by an engineered and evolved Clostridium thermocellum strain. Biotechnology for Biofuels 2014 7:75.

\section{Submit your next manuscript to BioMed Central and take full advantage of:}

- Convenient online submission

- Thorough peer review

- No space constraints or color figure charges

- Immediate publication on acceptance

- Inclusion in PubMed, CAS, Scopus and Google Scholar

- Research which is freely available for redistribution 